\title{
Cómo publicar en medicina de urgencia
}

\author{
Guadalupe López-Romero ${ }^{1}$, José J. Castillo-Pérez², Álvaro J. Montiel-Jarquin ${ }^{3}$, \\ Ma. Guadalupe Carrillo-Montes ${ }^{4}$, Sylvia C. Ramírez- Sánchez ${ }^{5}$ y Alejandro Villatoro-Martínez ${ }^{6 \star}$
}

${ }^{1}$ Servicio de Urgencias, Hospital General Dr. Gustavo Baz Prada, Instituto de Salud del Estado de México, Edo. Méx.; ${ }^{2}$ Área de Procesos digitalizados, Centro Médico Nacional Siglo XXI, Instituto Mexicano del Seguro Social, Ciudad de México; ${ }^{3}$ Division de Investigación en Salud, Unidad Médica de Alta Especialidad, Hospital de Especialidades, Centro Médico Nacional, Instituto Mexicano del Seguro Social, Puebla, Pue.; ${ }^{4}$ Área de Regulación, Centro Médico Nacional Siglo XXI, Instituto Mexicano del Seguro Social, Ciudad de México; ${ }^{5}$ Coordinación de programas de enfermería, Centro Médico Nacional Siglo XXI, Instituto Mexicano del Seguro Social, Ciudad de México; ${ }^{6}$ Área de Farmacovigilancia, Centro Médico Nacional Siglo XXI, Instituto Mexicano del Seguro Social, Ciudad de México. México

\section{Resumen}

Todo trabajo de investigación debiera ser publicado, sobre todo si es bueno, pero no siempre está garantizada su publicación. Escribir textos científicos requiere detalles en tiempo y trabajo. Al médico de urgencias durante su formación casi no se le enseña sobre investigación y publicación, además del desconocimiento de aspectos sobre edición y envío a una revista. El presente manuscrito se centra en fundamentos prácticos sobre cómo escribir un texto médico científico, indicando aspectos relevantes para su redacción, desde la concepción, redacción, metodología y análisis de la información para llegar a conclusiones adecuadas, lo que conlleva potenciar la posibilidad de publicación.

Palabras clave: Redacción. Artículo. Publicación. Rechazo.

\section{How to publish in emergency medicine}

\begin{abstract}
All research work should be attempted to be published even when it is not guarantied that this will occurre. Writing scientific texts requires time and work in detail in many regards. The emergency physician is almost never taught about research and the publishing of scientific work. Additionally, the lack of knowledge on editing and the process of submmiting this work to a scientific magazine. The current work focuses on practical knowkedge such as how to write a scientific text in medicine, indicating relevant aspects of composition; from its conception, edition, methodology and the analysis of information to reach adequate conclusions. All of the previous to increase the opportunities for a possible publication.
\end{abstract}

Key words: Writing. Article. Rejection. Publication.

\section{Introducción}

La redacción científica depende de un buen protocolo de investigación y la expectativa del autor o autores al redactarlo no garantiza su publicación, probablemente por falta de capacitación sobre redacción científica, que no es una habilidad innata, sino un aprendizaje continuo de investigadores e instituciones para formar

\section{Correspondencia:}

*Alejandro Villatoro-Martínez

E-mail: alexvillatorom @gmail.com
Fecha de recepción: 09-08-2020

Fecha de aceptación: 16-02-2021 DOI: 10.24875/REIE.20000034
Disponible en internet: 16-04-2021

Rev Educ Investig Emer. 2021;3(1):36-41 www.medicinadeemergencias.com 2604-6520 @ 2021 Sociedad Mexicana de Medicina de Emergencias, AC. Publicado por Permanyer México SA de CV. Este es un artículo open access bajo la licencia CC BY-NC-ND (http://creativecommons.org/licenses/by-nc-nd/4.0/). 
personal capacitado $0^{1,2}$. Esta destreza requiere paciencia y práctica constante ${ }^{3,4}$, es un aprendizaje diario ${ }^{5}$, donde cada autor perfecciona y desarrolla su estilo.

El presente trabajo presenta conceptos básicos para redactar un escrito científico desde su concepción, edición, revisión y publicación, considerando los errores para evitar su rechazo por la revista seleccionada y aumentando con ello la posibilidad de publicar. «Lo que se publica es permanente", exponiéndolo públicamente a la opinión del lector, del editor y de sí mismo.

\section{Previo al envío}

\section{Inicio a la redacción}

"Si tiene miedo y le tiembla la mano antes de escribir... ¡Felicidades! Está en el camino correcto». Rescribir, eliminar, borrar, toma tiempo; es un proceso difícil porque es tacharse a uno mismo, requiriendo entereza, fortaleza y mucha autoconfianza ${ }^{6}$.

Creemos que el proceso de redacción y escritura científica no se ha promocionado adecuadamente, por ello antes de iniciar debemos conocer los motivos para publicar $^{3,6,7}$ : a) crear currículo y tener una carrera académica ${ }^{7,8}$; b) publicar conocimientos nuevos e intercambiar ideas; c) satisfacción personal, y d) conseguir recursos para investigar, (patrocinios). Resumiendo, el publicar difunde los conocimientos y estimula el avance científico.

\section{Redacción}

Es como la práctica médica, «redactar bien para publicar... se debe realizar periódicamente para desarrollar y perfeccionar las destrezas necesarias". Las revistas no corrigen el contenido científico de los manuscritos, es responsabilidad de los autores que el escrito sea bien redactado $0^{9,10}$.

Frecuentemente se renuncia a escribir por considerar difícil crear escritos con calidad para ser aceptados. Desafortunadamente, el concepto «para escribir bien, hay que leer» no aplica, porque «hay profesionales que leen mucho, saben mucho y... escriben mal». Creemos que «él que hace ciencia también escribe ciencia»,10. Algunos conceptos antes de iniciar:

- La redacción debe ser estructurada y sucinta.

- Formar oraciones con menos de 30 palabras entre punto y punto.

- Usar palabras, frases y párrafos cortos, que formen ideas precisas ${ }^{8}$.
- Escribir como se habla (sin palabras rebuscadas, desconocidas o jerga coloquial) ${ }^{11}$.

- Ser preciso con el vocabulario, tecnicismos, sinónimos, conectores, etc.

- Usar citas, pero referidas correctamente... como lo solicita la revista.

Si es un artículo original se escribe en pasado; para acciones del pasado, pero que continúan en presente, se redactan en antepresente. Es «escribir claro, para que se entienda y corto para que lo lean» ${ }^{12}$. La retórica es de los políticos. Antes del envío hay que verificar que el texto sea coherente y conciso.

\section{El proyecto}

Elabore un plan que considere tiempo, recursos físicos, materiales y costo total del proyecto (incluyendo, si es el caso, el pago por publicación) ${ }^{7}$. Es recomendable escribir solo en lo que tenga experiencia, apoyado con bibliografía actual, además de consultar recomendaciones y actualizaciones para envío en el International Committee of Medical Journal Editors $(\text { ICMJE })^{13}$.

\section{Eligiendo la revista}

Si su proyecto está terminado y con resultados, solo deberá buscar la revista en la que desea publicar, donde su escrito sea del interés de los editores. Si no es el caso, considerar dónde se enviará el escrito, examinando: prestigio, factor de impacto, porcentaje de aceptación, audiencia, política editorial y tiempos de gestión. Para aceptar manuscritos las revistas priorizan el tema, el número de páginas y la calidad ${ }^{3,14}$. Seleccionarla aumenta la posibilidad de publicación, siempre y cuando se adhiera a las normas para autor de la revista, pudiendo redactar de nuevo para adaptarlo al formato solicitado. Considere en el envío (carta de presentación del escrito, información de autores, tipo de manuscrito y cesión de derechos).

\section{Sobre el escrito}

\section{Título}

Fomenta el interés del manuscrito, es lo primero que se lee ${ }^{5}$. Debe ser claro para editor, revisores y lectores, «usando el menor número de palabras para escribirlo», sin subtítulos o abreviaturas. Se busca mediante palabras clave ${ }^{15} \mathrm{o}$ algoritmos de búsqueda (Google). 


\section{Resumen y abstract}

Se redacta en pasado, sin abreviaturas, referencias o datos estadísticos, tablas o figuras. Hay que exponer el máximo de ideas con el mínimo de palabras. «Un buen artículo es precedido de un buen resumen, si este es pobre solo anuncia inconsistencias y errores". Un mal resumen no permite segundas oportunidades ${ }^{14}$. Es una síntesis de nuestro estudio e incluye: por qué (antecedentes), el planteamiento (objetivos), cómo fue realizado (material y métodos), hallazgos (resultados) y su interpretación y consecuencias (conclusiones) ${ }^{16}$. Debe tener un máximo de palabras (200-300 como promedio) ${ }^{17,18}$. «Debe contener exactamente lo que presentará el manuscrito, en un lenguaje simple»19,20.

El abstract es resumen en inglés y también se redacta en pasado. La revista Annals of Botany lo ejemplifica muy bien ${ }^{13}$ : a) background and aims, b) methods, c) key results y d) conclusions. Otra forma sería: a) background, b) scope y c) conclusions, útil para revisiones, reportes breves y artículos técnicos.

\section{Palabras clave o key words}

Las solicita la revista ${ }^{21}$. Serán de 3 a 10, idealmente mediante descriptores (MeSH, Medical Subject Headings). Su objetivo es homologar y asegurar la búsqueda, visualización y citación del escrito ${ }^{15}$. Con ello lectores y editores verifican si el artículo es de su interés, permiten a las bases de datos e índices la localización rápida y eficiente de artículos, agrupándolos por temas, además de ubicar revisores para enviar el manuscrito a evaluación ${ }^{19}$.

\section{Abreviaturas}

Solo las utilizadas en el escrito, evítelas a menos que sea totalmente necesario ${ }^{22}$.

\section{Introducción}

Orienta y proporciona al lector la información para ubicar el artículo en el contexto de lo que se ha escrito, situándolo en el presente; va de lo general a lo particular. Para redactarla deberá seleccionar cuidadosamente las palabras que expliquen el trabajo, respondiendo a la pregunta ¿cuál es el problema investigado? ${ }^{23}$. Incluirá: a) la naturaleza y alcance, b) la revisión de la literatura sobre el tema, y c) la descripción de los resultados previos (si es pertinente). El planteamiento del problema, la pregunta de investigación, los objetivos e hipótesis en un artículo no se describen explícitamente, sino implícitamente (dentro de esta sección).

\section{Planteamiento del problema}

Expresa claramente las acciones que responderán a la pregunta de investigación, expresando la relación entre variables ${ }^{22}$. Su extensión será breve.

\section{Pregunta}

Será clara y concreta. Permite elegir el diseño de estudio y debe cumplir con cinco premisas: que sea interesante, factible, novedosa, ética y relevante ${ }^{24}$.

\section{Hipótesis}

Permitirá identificar cuál será el posible resultado del proyecto. Muy importante en diseños analíticos y experimentales, indica los resultados que se desea obtener y establece las bases de las pruebas estadísticas requeridas para analizar los datos y su nivel de significancia.

\section{Objetivos}

Son las tareas que realizar por el investigador para concretar y especificar el alcance del proyecto, fundamentan la metodología. El general indica lo que se pretende alcanzar con el trabajo de investigación y los específicos responden a aspectos concretos o etapas del proyecto de investigación, son tareas que realizar para cumplir el objetivo general.

\section{Métodos}

Se redactan en pasado, describiendo ¿qué y cómo se hizo? Se inicia con el tipo de población del estudio y se incluyen detalles sobre marca, características y especificaciones técnicas del equipo, recursos y material utilizado. Si el estudio es en humanos, se indicarán los criterios de inclusión y exclusión, con las respectivas autorizaciones de los Comités de Investigación y de Ética. Se indica cómo se cumplió con las normas de buena práctica clínica y de laboratorio, cómo se controlaron los sesgos y el cálculo del tamaño de muestra. Debe ser directa y precisa e incluir la siguiente información ${ }^{23}$ :

- Diseño: tipo de estudio realizado (observacional, experimental, serie de casos, etc.). 
- Población: criterios de selección (aleatoria, sistemática o no probabilística).

- Entorno: dónde se realizó el estudio (hospital, laboratorio, etc.).

- Intervención: describa tratamientos, mediciones y software utilizado.

- Análisis estadístico: explique el método y cómo se analizaron los datos.

Los instrumentos empleados deben ser validados, indicando cómo se obtuvieron, si no, convendrá explicar cómo se realizó la validación.

\section{Análisis estadístico}

Incluye la recogida, organización, síntesis y análisis de datos mediante estadística descriptiva y el empleo de alguna medida de asociación de riesgo. Para protocolos analíticos, es necesario emplear estadística inferencial, explicar las pruebas de contraste de hipótesis que se van a utilizar y el nivel de significancia estadística $^{25,26}$.

\section{Resultados}

Se redactan en pasado, considerando en su presentación texto, tablas y figuras. Las características demográficas más representativas de los participantes en el estudio se pueden concentrar en una tabla de forma clara, concisa y objetiva. Del análisis estadístico deberá seleccionar la información más relevante. Los resultados se ligan a los métodos utilizados, cada método referido tendrá un resultado que responda a las preguntas planteadas ${ }^{22}$.

No incluirá referencias bibliográficas y menos la interpretación personal de los datos. Si son extensos recomendamos la representación con gráficas y figuras $^{27}$. Evite errores obvios, por ejemplo, «respuesta a tratamiento $33.3 \%$, sin efecto $33.3 \%$, efecto moderado 33.3\%".

\section{Gráficas y tablas}

Facilitan la explicación del escrito y resultados con orden. Debe presentarse una sola idea de fácil lectura, con información ordenada, las variables en filas y otros elementos en columnas. Es preciso evitar las frases «en la tabla inferior» 0 «la siguiente». Hay que cuidar la alineación con el texto, el tipo y tamaño de letra, los márgenes y la separación de columnas. Tablas, cuadros y gráficos deberán guardarse por separado en archivos con su propio nombre o numeración.

\section{Discusión}

Se hace en oraciones cortas con buen uso de lenguaje para demostrar la validez del proyecto, evite conceptos vagos; no utilice la «técnica del calamar: esconder en tinta los hallazgos». Una buena discusión incluye:

- Demostrar las relaciones principales de las variables y el alcance de los resultados.

- Comparar los resultados con otros similares e interpretar sus hallazgos.

- Discutir las implicaciones del trabajo y repercusiones.

- Cuidar que cada aspecto presentado esté demostrado en los resultados del trabajo.

Si se requiere podrá incluir parte del análisis estadístico en la discusión, presentando las relaciones entre hechos observados con los resultados, esto justifica las conclusiones a las que se llegó. La discusión expone lo programado y demostrado mediante hechos contrastados.

\section{Conclusiones}

Son directas y simples, sintetizan las ideas cardinales del estudio, dan el punto de vista de los autores, representan una verdad (lo investigado). Pero no presenten conclusiones sobre hechos no comprobados, ya que indican desconocimiento del tema y el rechazo del escrito.

\section{Citas y referencias}

Las citas son la forma abreviada de referencia, permiten la identificación de un documento, dan sustento académico al manuscrito. Constituyen un elemento básico e insustituible, apegándose a las normas para autores de la revista (Vancouver, APA o Harvard según se soliciten). Deben incluir los artículos publicados en los últimos cinco años.

\section{Financiamiento}

De haber recibido financiamiento se indicará el nombre de la institución pública o privada, tipo de participación en el estudio y número de subvención.

\section{Conflicto de interés}

La publicación de un artículo en una revista puede tener implicaciones personales, financieras e institucionales, afectando o favoreciendo a autores, 
instituciones, empresas y países. De existir un potencial conflicto, debe ser declarado de forma transparente, para que se conozca, evalúe y exponga en la publicación, sin implicar su aceptación ${ }^{26}$.

\section{Consideraciones antes del envío}

\section{Después de escribir}

Terminado el escrito y antes del envío se debe revisar el texto para evitar errores ocultos durante la escritura (ceguera del autor). Otra forma de búsqueda de fallas es solicitar la ayuda a un tercero que juzgue objetivamente el escrito, que aporte comentarios para mejora y así entregar un trabajo de calidad y aumentar la aceptación.

\section{Autoría e institución}

Antes del envió del documento final debe definirse el orden de los autores, principalmente de dos: primer autor y corresponsal, lo solicita la revista y permite conocer la participación de cada autor. Vigile que su nombre esté correctamente escrito. Facilita nuestro reconocimiento nacional e internacional, debido a que en algunos países solo se utilizan el nombre y primer apellido (anglosajones), y evita que sus resultados se pierdan. Considere utilizarlos unidos por un guion, no olvide que el nombre de la institución, hospital, servicio y unidad donde labora tienen nombre propio; no es conveniente traducirlos, podría perder visibilidad como autor, institución y para el país ${ }^{28,29}$.

\section{Aceptación o rechazo}

Cuando se envía un artículo a publicar se desea comunicar «algo" que fomente el avance de la ciencia, "debe seleccionar la revista adecuada». Pero aun con ello y a pesar de que se sigan todos los lineamientos, no todos se aceptan (porcentaje de aceptación o rechazo de la revista).

La revista evalúa: 1) tipo de estudio; 2) fluidez de redacción; 3) la metodología, hipótesis, objetivos, métodos empleados, tamaño de la muestra y tipo estadística empleada; 4) para los resultados, se evalúa su obtención y concordancia con los objetivos; presentación e interpretación de tablas y figuras; 5) las limitaciones del estudio, datos negativos y hallazgos inesperados; 6 ) las conclusiones finales, sustentadas en resultados su inferencia y generalización futura, y 7) las citas, bibliografía que sea reciente y con calidad, trabajos previos del autor que avalen su conocimiento del tema. Posterior a ello hay cuatro posibles escenarios de su manuscrito que el editor le informará: a) aceptación del escrito sin modificaciones (muy rara); b) aceptación con modificaciones (la más común); c) reenvío para revisión y modificaciones (se acepta, solo si se realizan), y d) no publicable (según el porcentaje de aceptación de la revista). Todas tienen fecha de cumplimento para hacer los cambios si no se rechaza ${ }^{14}$.

\section{Conclusiones}

Difundir los resultados de una investigación requiere perseverancia, conocer el tema y apegarse a lo que solicita una revista para su aceptación, pero siempre deja una satisfacción ver que nuestro esfuerzo puede aportar conocimiento.

\section{Financiamiento}

La presente investigación no ha recibido ninguna beca específica de agencias de los sectores públicos, comercial, o sin ánimo de lucro.

\section{Conflicto de intereses}

Los autores declaran no tener conflicto de intereses alguno.

\section{Responsabilidades éticas}

Protección de personas y animales. Los autores declaran que para esta investigación no se han realizado experimentos en seres humanos ni en animales.

Confidencialidad de los datos. Los autores declaran que en este artículo no aparecen datos de pacientes.

Derecho a la privacidad y consentimiento informado. Los autores declaran que en este artículo no aparecen datos de pacientes.

\section{Bibliografía}

1. Barrios-de Tomasi J, Varela-Carlos ME. Diez simples consejos para escribir un artículo científico. Rev Sal Quintana Roo. 2015;8:24-5.

2. Corcelles M, Cano M, Mayoral P, Castelló M. Enseñar a escribir un artículo de investigación mediante la revisión colaborativa: Percepciones de los estudiantes. Rev Signos. 2017;50:337-60.

3. Contreras A, Ochoa Jiménez R. Manual de redacción científica: Escribir artículos es fácil, después de ser difícil -Una guía práctica-. México D.F., México: Ediciones de la Noche; 2010.

4. Sanesteban Echarri O, Nuñes Morales NI. Cómo escribir un artículo científico por primera vez. Psiquiatr Biol. 2017;24:3-9.

5. Rubalcaba E. 52 tips para escribir claro y entendible. México D.F., México: Lectorum; 2011. pp. 13-147.

6. Kotsis SV, Chung KC. A guide for writing in the scientific forum. Plast Reconstr Surg. 2010;126:1763-71. 
7. Earnshaw JJ. How to write a clinical paper for publication. Surgery (Oxford). 2012;30(9):437-41.

8. Kamat P. How to write an effective how to write an effective research paper [Internet]. Prashant V. Kamat, University of Notre Dame, Department of Chemistry \& Biochemistry and Radiation Laboratory [consultado: 20 de julio de 2019]. Disponible en: http://www3.nd.edu/ pkamat/pdf/ researchpaper.pdf

9. Zwaaf E. '8 reasons I accepted your article' [Internet]. Elsevier Connect; enero de 2013 [consultado: 22 de junio de 2019]. Disponible en: https:// www.elsevier.com/connect/8-reasons-i-accepted-your-article

10. Gutiérrez- Díaz M, Santamaría-Pablos A, García Benaite D, Figuero Redondo C. Razones para investigar: motivación y reconocimiento. SEMERGEN. 2007;33:111-2.

11. Griffies SM, Perrie WA, Gaëlle Hull. Elements of style for writing scientific journal articles [Internet]. Elsevier; 2013 [consultado: 24 de julio de 2019]. Disponible en: https://www.gfdl.noaa.gov/wp-content/ uploads/2018/08/Elements_of_Style.pdf

12. Jiménez-Serrano M. Las 7 llaves de la escritura: claves profesionales para redactar bien [Internet]. El confidencial, Sección Alma, corazón y vida; octubre de 2013 [consultado: 1 de agosto de 2019]. Disponible en: https://www.elconfidencial.com/alma-corazon-vida/2013-10-03/las-7-Ilaves-de-la-escritura-claves-profesionales-para-redactar-bien_31587/

13. Preparing a Manuscript for Submission to a Medical Journal [Internet]. International Committe of Medical Journal Editors [consultado: 5 de agosto de 2018]. Disponible en: http://www.icmje.org/recommendations/browse/manuscript-preparation/preparing-for-submission.html

14. Marin-Garcia JA. ¿Por qué los revisores rechazan mi artículo si era tan maravilloso? WPOM. 2011:2:7-10.

15. Fernández-Altuna MA, Martínez del Prado A, Arriarán-Rodríguez E, Gutiérrez Rayónc D, Toriz-Castillo HA, Lifshitz Guinzberg A. Uso de los MeSH: una guía práctica. Inv Ed Med. 2016;5:220-9.

16. European Association of Science Editors. EASE Guidelines for Authors and Translators of Scientific Articles to be published in English [Internet] European Association of Science Editors; diciembre de 2015 [consultado: 7 de agosto de 2019]. Disponible en: http://www.ease.org.uk/wp-content uploads/ease_guidelines-2015.pdf
17. Abstract Guidelines for Papers [Internet]. Academic Conferences International [consultado: agosto de 2019]. Disponible en: http://www.academic-conferences.org/policies/abstract-guidelines-for-papers/

18. Silva Hernández D. El resumen del artículo científico de investigación y recomendaciones para su redacción. Rev Cubana Salud Pública. 2010;36;179-83.

19. Heredia A. ¿Por qué y cómo publicar artículos científicos en revistas internacionales? Seminario para Autores [Internet]. España: Control, Electrónica, Modelado y Simulación (CEMOS), Universidad Industrial de Santander (UIS); 2011 [consultado: 1 de septiembre de 2019]. Disponible en: https://www.uis.edu.co/webUIS/es/index.jsp

20. Bahrani T, Soltani R. Why not reflect the outcome of your study in an academic paper? J Educ Pract. 2012:3:1-5

21. Moncada-Hernández SG. Cómo realizar una búsqueda de información eficiente. Foco en estudiantes, profesores e investigadores en el área educativa. Inv Ed Med. 2014;3:106-15.

22. African Federation for Emergency Medicine. Author Information Pack. AfJEM. 2016:1-14

23. Mateu Arrom L, Huguet J, Errando C, Breda A, Palou J. Como escribir un artículo original. Actas Urol Esp. 2018;42:545-50.

24. Miranda-Novales M, Vilasís-Keever. El protocolo de la investigación. Parte I: la población de estudio. Rev Aler Méx. 2016;63:201-6.

25. Vargas Azofeifa D. Manejo instrumental del concepto de hipótesis en el diseño de un proyecto de investigación. Gestión. 2006;14(2).

26. Hernández Sampieri R. Metodología de la investigación. Las rutas cuantitativa, cualitativa y mixta. México, D.F.: McGraw-Hill; 2018. pp. 338-343.

27. Salas PS. Conflicto de intereses en la investigación biomédica. Rev Chil Obstet Ginecol. 2010;75:143-5

28. Bravo-Vinaja A. Sugerencias para la normalización de los nombres de autores de habla hispana. Rev Fitotec Méx. 2014:37:3-4.

29. Propuesta de manual de ayuda a los investigadores españoles para la normalización del nombre de autores e instituciones en las publicaciones científicas [Internet]. Fundación Española para la Ciencia y la Tecnología [consultado: 1 de agosto de 2019]. Disponible en: https://www.unavarra. es/digitalAssets/186/186628_100000normalizacion_nombre_autor.pdf 